\title{
Matlab tool REGCONT2: effective source depth estimation by means of Tikhonov's regularized downwards continuation of potential fields
}

\author{
Roman PAŠTEKA, David KUŠNIRÁK, Roland KARCOL \\ Department of Applied and Environmental Geophysics, \\ Faculty of Natural Sciences, Comenius University, \\ Mlynská dolina, Ilkovičova 6, 84248 Bratislava, Slovak Republic; \\ e-mail: roman.pasteka@uniba.sk
}

\begin{abstract}
Transformation based on downward continuation of potential fields is an important tool in their interpretation - depths of shallowest important sources can be determined by means of stable downward continuation algorithms. We analyse here selected properties of one from these algorithms (based on Tikhonov's regularization approach) from the scope of two most important discretization parameters - dimensions of the areal coverage of the interpreted field and the sampling interval size. Estimation of the source depth is based on the analysis of computed $L_{P}$-norms for various continuation depths. A typical local minimum of these norms disappears at the source depth. We show on several synthetic bodies (sphere, horizontal cylinder, vertical rod) and also real-world data-sets (results from a magnetic survey for unexploded ordnance detection) that there is a need for relatively large surroundings around the interpreted anomalies. Beside of this also the sampling step plays its important role - grids with finer sampling steps give better interpretation results, when using this downward continuation method. From this point of view, this method is more suitable for the interpretation of objects in near surface and mining geophysics (anomalies from cavities, unexploded ordnance objects and ore bodies). Anomalies should be well developed and separable, and densely sampled. When this is not valid, several algorithms of interpolation and extrapolation (grid padding methods) can improve the interpretation properties of studied downward continuation method.
\end{abstract}

Key words: gravimetry, magnetometry, interpretation, analytical continuation

\section{Introduction}

Transformations of potential fields in applied geophysics (magnetic, gravity) belong among important tools in their processing and interpretation. 
These transformations, in general, do not directly determine field sources, but they can often provide insights that help to understand the natures of sources (Blakely, 1995, p. 311). Analytical continuation in source free area is one of the most important procedures among them. In general, we usually speak about upward continuation (UC, in direction away from the sources) and downward continuation (DC, in the direction closer to the sources). Downward continuation is an important procedure in potential fields interpretation - it can be utilized for two main purposes (Mudretsova and Veselov, 1990a, p. 328) approximation of the interpreted field on: a) depth level, which is closer to the sources and b) estimation of the sources positions/depths. On the other hand, it is an instable operation (amplifying noise and errors in data) and it must be treated in a special way.

This contribution is focused on the DC transformation as a tool for sources depths estimation in applied geophysics. It tries to present some of important properties of DC method from the aspect of its practical application. From the theoretical point of view, potential fields described by continuous functions and defined on domains with infinity dimensions, can be continued in the source free area down to the upper boundary of the shallowest source (Baranov 1975, p. 32-33). But in the real world these two important conditions are not valid: our data are not continuous (we work with discrete digital datasets) and they are not defined on areas with infinite dimensions (our exploration areas are always limited). Accepting this, we should analyse the properties of stable DC algorithms from the scope of two most important discretization parameters - dimensions of the areal coverage of the interpreted field and the sampling interval size. This is valid for profile data and also areal data-sets (grids). We show the important role of these two parameters on the results of used DC algorithm (focused on source depth estimation), presented on several synthetic model studies and real-world datasets interpretation (magnetic survey for UneXploded Ordnance detection). Together with our analysis we present here a software solution of all the discussed aspects - a Matlab script REGCONT2, which is an upgrade of our previously published program REGCONT (Pašteka et al., 2012). It can be used for an effective determination of sources depths in applied gravimetry and magnetometry (Matlab script is free for academic and scientific use). 


\section{Tikhonov's regularization approach in stable downward continuation}

Downward continuation of potential fields is an instable operation due to its inherent properties as a high-pass filter (in Fourier domain the spectral characteristic of this operation is an exponential function of the wave-number). From practical point of view it means that any error and/or noise (in the original data) will be strongly boosted during this transformation. This is valid also for the edge effect (Gibb's phenomenon), which then often flows into strong artificial oscillations in the DC data-sets (see e.g. the Fig. 1 in Pašteka et al., 2012). These disturbances (oscillations) can even mask the field anomalies itself. There have been published several interesting methods for finding a stable solution of this problem (e.g. Fedi and Florio, 2002; Trompat et al., 2003; Cooper, 2004) and during the last years there can be followed something like a "boom" in this scientific area (e.g. Ma et al., 2013; Zeng et al., 2013; Zhang H. et al., 2013; Abedi et al., 2014; Zeng et al., 2014; Zeng et al., 2015; Zhang Y. et al., 2016, Zhou et al., 2018; Florio and Fedi, 2018; Zhang et al., 2018).

In our contribution we work with the classical approach from Tikhonov et al. (1968), using the regularization approach in Fourier wavenumber domain (exact derivation of the spectral characteristics of the regularized downward continuation is given in the Appendix). Some authors use for this approach the abbreviation TRDC method (Tikhonov Regularized Downward Continuation; Zhang H. et al. 2013) or simply TR method (Tikhonov Regularization; Zhang $Y$. et al., 2016). Main idea of this approach is to use a special filter form (Eq. A.6) in the wavenumber domain, which combines the classical downward continuation spectral characteristics (exponential function) multiplied with a low-pass filter. Properties of this low-pass filter are controlled by the value of regularization parameter $\alpha$, where the lower values mean lower role of regularization, higher values cause intensive damping of the high spectral content of the downward continued data. The classical challenge (as in other regularization methods) is to find an optimum value of the regularization parameter $\alpha$, for which some kind of equilibrium between the original nature of the transformation (DC) and the smoothing effect of the low-pass filter occurs. In the traditional Tikhonov's method, C-norms of the solutions for different values of regularization parameter $\alpha$ have been constructed and interpreted (Tikhonov et al., 1968; Glasko et al., 1970). 
From functional analysis we know that the C-norm is equal to $\mathrm{L}_{\infty}$-norm. These norms compare two adjacent solutions (obtained for two adjacent values of regularization parameter) and plot their distance (C-norm) as a function of the regularization parameter $\alpha$. This is plotted as a log-log graph with the regularization parameter $\alpha$ on the horizontal axis and the C-norm value on the vertical axis. These norms have always been constructed for a large interval of regularization parameter $\alpha$. In most of the cases are the values of $\alpha$ ranging from $10^{-20}$ to $10^{+20} \mathrm{~m}^{-1}$, in a geometrical sequence with usual factor of 1.1. Their typical shape is convex and positive with the existence of a local minimum in the vicinity of their global maximum. This local minimum reports on the occurrence of the mentioned equilibrium and the optimum regularization parameter value is bound to this local minimum. This idea is very close to the concept of L-curve (Lawson and Hanson, 1974; Hansen, 2007), which is used in many regularization methods in mathematical physics. Appearance and disappearance of the local minimum can be used for the source depth estimation in the TRDC method - vanishing of this local minimum during downward continuation to larger depth levels points to the depth of the source (e.g. Tikhonov et al., 1968; Glasko et al., 1970; Pašteka et al., 2011, 2012). We can say in other words: when we enter with the continuation depth into a non-harmonic space (into the sources), method cannot find a correct solution (local minimum vanishes). Of course that the source depth depends in this method on the source shape - for vertically elongated bodies it is the top, for isometric bodies it is the centre. Our small contribution to the classical TRDC method was the way that we construct beside the classical C-norm also other $\mathrm{L}_{\mathrm{P}}$-norms (Pašteka et al., 2012). We usually calculate $\mathrm{L}_{2}$-norms, $\mathrm{L}_{1}$-norms and $\mathrm{L}_{0.5}$-norms and then we select that type of norm, where the local minima are well developed (with sharper side parts of curves). It is interesting that in some situations give these other $\mathrm{L}_{\mathrm{P}}$-norms better results than the classical $\mathrm{C}$-norms. At the moment, we are not able to scientifically explain the reason, why some kinds of norms work better in some situations - we simply try all of them and then we select the best one.

In Fig. 1 we have displayed results for the depth estimation of two simple synthetic models - a 2D horizontal cylinder with circular cross-section (depth of the cylinder centre is $1000 \mathrm{~m}$ ) and a sphere (depth of the sphere centre is $5 \mathrm{~m}$ ). The step in DC was for the case with horizontal cylinder 
$200 \mathrm{~m}$ and in the case with sphere $1 \mathrm{~m}$. In the graphs of displayed C-norms (Fig. 1b and 1e), a nicely developed local minima are visible (with sharp side parts of curves) and detected local minimum disappear exactly at corresponding DC depths, where the most important source points of both bodies are positioned (centres of them). In Fig. 1c and $\mathrm{f}$ it is shown (with a detailed step in DC) that the disappearance occurs on depths, which are very close to the source position - method can focus on these details in a very detailed way - any reasonable small step of the depth change can be selected. But to speak here about some kind of the depth resolution is not appropriate, because the precision of the method depends on discretization parameters of the interpreted field (as it will be given later). This example belongs to the so-called "text-book" cases - everything works perfectly here. Why? Because the interpreted synthetic fields were evaluated
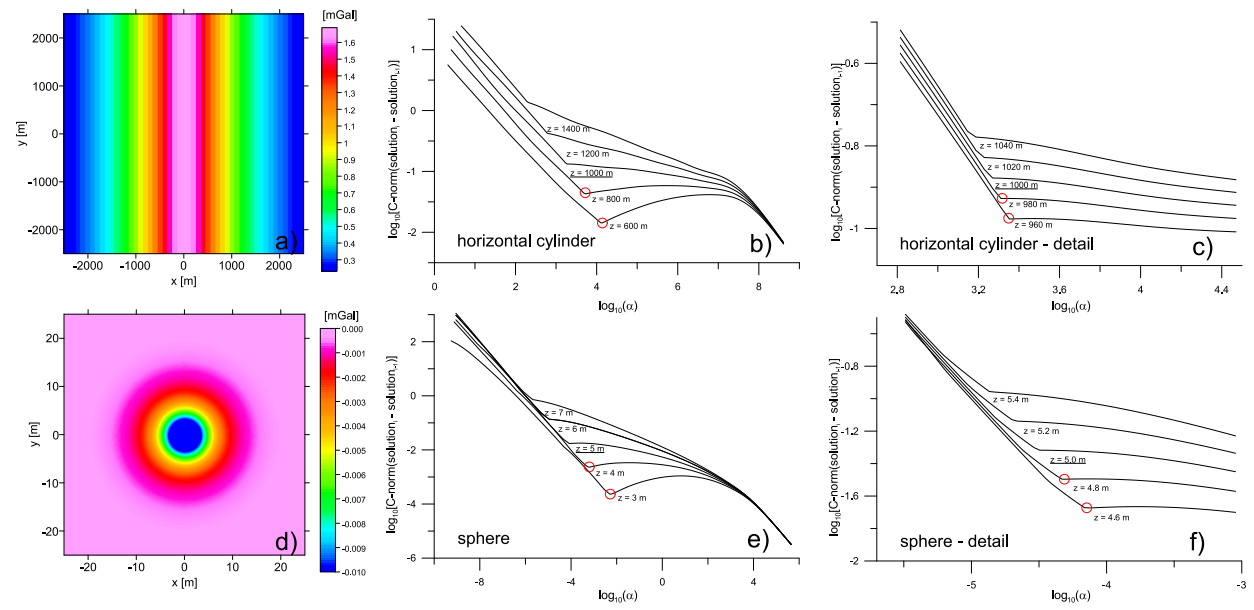

Fig. 1. Results of depth estimation for 2D horizontal cylinder and sphere. a) Synthetic vertical component of gravitational acceleration field $\left(\mathrm{V}_{\mathrm{z}}\right)$ for the horizontal cylinder in the depth of $1000 \mathrm{~m}, \mathrm{~b}$ ) C-norms obtained from the depth estimation of horizontal cylinder depth (depths from 600 to $1400 \mathrm{~m}$, step $=200 \mathrm{~m}$ ), c) C-norms for depths close to the source position (depths from 960 to $1040 \mathrm{~m}$, step $=20 \mathrm{~m}$ ), d) synthetic vert. component of gravitational acceleration field $\left(\mathrm{V}_{\mathrm{z}}\right)$ for the sphere in the depth of $5 \mathrm{~m}$, e) C-norms obtained from the depth estimation of sphere depth (depths from 3 to $7 \mathrm{~m}$, step $=1 \mathrm{~m}), \mathrm{f}$ ) C-norms for depths close to the source position (depths from 4.6 to $5.4 \mathrm{~m}$, step $=0.2 \mathrm{~m}$ ). Sampling steps of the input grids are in the case of the cylinder: $\Delta x=\Delta y=100 \mathrm{~m}$, in the case of the sphere: $\Delta x=\Delta y=0.5 \mathrm{~m}$. Density of the $2 \mathrm{D}$ cylinder: $+1000 \mathrm{~kg} \mathrm{~m}^{-3}$, density of the sphere: $-1000 \mathrm{~kg} \mathrm{~m}^{-3}$. 
on an area with satisfactory large dimensions (its length in both directions is 5 times the source depth) and the anomalous field is properly sampled (anomaly half-width is given by tens of grid points). But what will happen, when these two important aspects (anomalous field areal dimensions and sampling step) will not have such "ideal" values? We can see a representative example in Fig. 2 (slightly inclined sub-vertical rod with the top in $10 \mathrm{~m}$ depth): in the case of under-sampled data with small areal dimensions (Fig. 2c and 2d) the estimated source depth is incorrect - it is too shallow
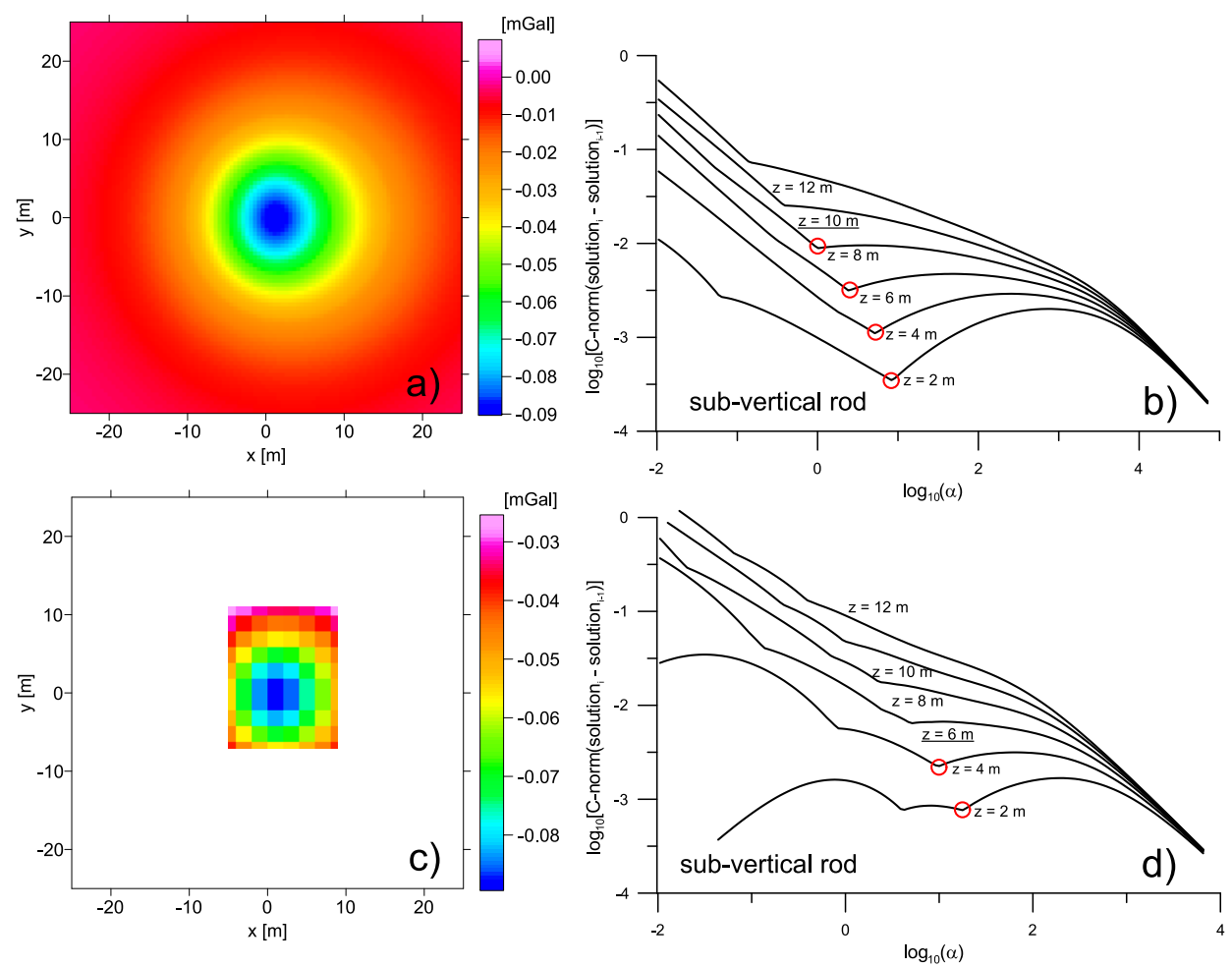

Fig. 2. Results of depth estimation for an inclined sub-vertical rod with the top in the depth of $10 \mathrm{~m}$ - a "good and bad" example: a) Synthetic vertical component of gravitational acceleration field $\left(\mathrm{V}_{\mathrm{z}}\right)$, calculated on larger area $(50 \times 50 \mathrm{~m}$ size $)$ with small sampling step: $\Delta x=\Delta y=0.5 \mathrm{~m}, \mathrm{~b})$ corresponding C-norms obtained from the depth estimation, c) synthetic vert. component of gravitational acceleration field $\left(\mathrm{V}_{\mathrm{z}}\right)$, calculated on small area $(10 \times 10 \mathrm{~m}$ size $)$ with large sampling step: $\Delta x=\Delta y=2 \mathrm{~m}$, b) corresponding C-norms obtained from the depth estimation. Density of the inclined sub-vertical rod: $-2000 \mathrm{~kg} \mathrm{~m}^{-3}$. 
(6 m instead of correct $10 \mathrm{~m}$ ). Shapes of the C-norms in Fig. $2 \mathrm{~d}$ are not well developed and these graphs do not possess the necessary sharp minima in their shapes. For a comparison we give also a result for well sampled field with large dimensions (Fig. 2a and 2b) - here the corresponding C-norms are well developed and the source depth $(10 \mathrm{~m})$ is correctly estimated.

We had experienced not well developed $\mathrm{C}$-norms and other $\mathrm{L}_{\mathrm{P}}$-norms in various situations and were wondering what could be the reason of that. Therefore, we started a set of experiments with these two important parameters - dimensions of the field area and its sampling steps. Results of these experiments will be shown later in this paper.

\section{Software realization of TRDC method for source depths estimation - Matlab script REGCONT2}

For the practical use of the proposed TRDC algorithm we have developed a Matlab script REGCONT, which was published together with our paper Pašteka et al. (2012). This script is suitable for UC and DC transformation of profile and grid potential data, but it is quite cumbersome for source depth estimation - user had to evaluate the corresponding $\mathrm{L}_{\mathrm{P}}$-norms for each depth of continuation and then plot all these output norms separately in some another software (or in Matlab) with the aim to detect the continuation depth, where the local minimum in analysed $\mathrm{L}_{\mathrm{P}}$-norms disappears. From this reason, we have decided to develop a new feature called "Source Depth Estimation" tool (SDE), which became part of the new version REGCONT2. Beside this, we have updated the main computational core of the script into a vector form, which significantly speed up the script. User can choose within the SDE tool the type of used $\mathrm{L}_{\mathrm{P}}$-norm $\left(\mathrm{C}\right.$-norm, $\mathrm{L}_{2}$-norm, $\mathrm{L}_{1^{-}}$ norm or $\mathrm{L}_{0.5}$-norm) and parameters of the downward continuation process: minimal depth, maximal depth and the step of depth change (see Fig. 3). After the realization of all calculations, selected norms are plotted for all continuation depth levels and in the norm graphs local minima are automatically detected and marked by a red circle. Position of the local minimum is determined by means of a simple 3-point operator, which starts on each plotted norm from the left-hand edge of the graph. Proposed Matlab script REGCONT2 is free for academic and scientific use and it can be downloaded together with supporting files and a user manual from the server: 
http://www.kaeg.sk/vyskum/projekt-vega-2014/project-vega-1-0462-16/.

In the next part of our paper we analyse on a simple synthetic model (a sphere) discussed important numerical aspects of interpreted fields (anomalous field areal dimensions and sampling step size) and give also suggestions, how to solve problems in cases, when these parameters are not sufficient. Further we present few experiences with real world data-sets (magnetic surveys for UneXploded Ordnance detection).

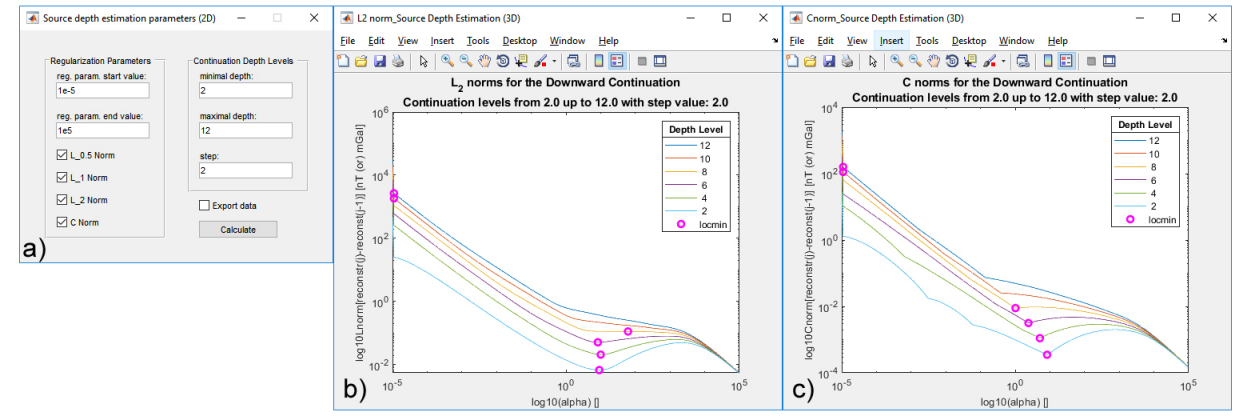

Fig. 3. Working environment of the "Source depth estimation" tool application in the frame of REGCON2 Matlab script. a) Selection of $\mathrm{L}_{\mathrm{P}}$-norms and continuation depths, b) displayed $\mathrm{L}_{2}$-norms, c) displayed C-norms. Small circles in graphs of displayed norms represent automatically detected local minima in their shapes.

\section{Synthetic model - sphere in gravimetry}

We demonstrate the discussed properties of the classical Tikhonov's regularized downward continuation (TRDC) algorithm on the anomalous gravitational field of a sphere (depth of its centre $=1000 \mathrm{~m}$, radius $=200 \mathrm{~m}$, density contrast $=1000 \mathrm{~kg} \mathrm{~m}^{-3}$ ) for various dimensions and sampling intervals of the downward continued fields. Our first trials were focused on the influence of the sampling size of the grid element (this is valid also for profile step). In general, it is valid that interpreted anomalies have to be well sampled - at least with 10 (and more) points within the half-width of the anomaly. We can see in Fig. 4a and $4 \mathrm{~b}$ that for small sampling steps $(\Delta x=\Delta y=100 \mathrm{~m}$, $\Delta x=\Delta y=200 \mathrm{~m}$ ), displayed C-norms have very well developed sharp local minima, which disappear at the correct depth $=1000 \mathrm{~m}$. This is not the 
case for larger sampling steps $(\Delta x=\Delta y=250 \mathrm{~m}, \Delta x=\Delta y=500 \mathrm{~m})$ in Fig. $4 \mathrm{c}$ and $4 \mathrm{~d}$ - local minima are not well developed and cannot be therefore used for depth estimation. Such a behaviour was realized also in the case of other simple geometrical shapes, as horizontal cylinder and vertical rod (not shown in this paper). It seems that this could be "a deep hit" to the TRDC method, but there exist a relatively easy solution (numerical) for this problem - interpolation. With relatively simple interpolation methods, offered in used commercial software (e.g. Golden Software Surfer, Matlab) we can re-grid the original dataset to a more dense grid and try to apply the TRDC method anew (with displaying several types of $\mathrm{L}_{\mathrm{P}}$-norms). From our experiences, the well-known Kriging (Cressie, 1991) and Minimum Curvature methods (Smith and Wessel, 1990) give the best results. In Fig. 5 we can see results from an experiment, where we took the grid from Fig. $4 \mathrm{~d}$ (with parameters $\Delta x=\Delta y=500 \mathrm{~m}$ ) and have re-gridded it to new grids
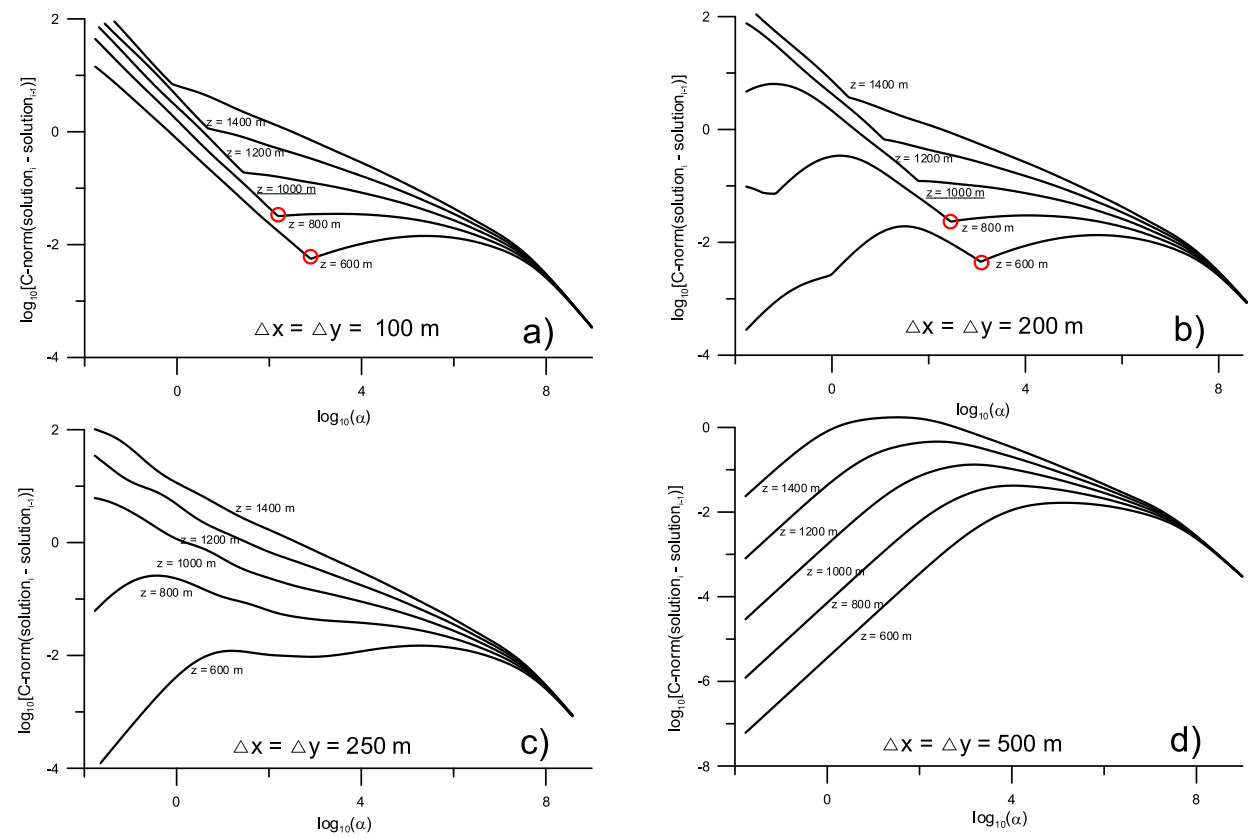

Fig. 4. Graphs of evaluated C-norms for the sphere model (grid dimensions for all cases: $5 \times 5 \mathrm{~km}$ ) calculated for different sampling steps: a) $\Delta x=\Delta y=100 \mathrm{~m}$, b) $\Delta x=\Delta y=$ $200 \mathrm{~m}, \mathrm{c}) \Delta x=\Delta y=250 \mathrm{~m}, \mathrm{~d}) \Delta x=\Delta y=500 \mathrm{~m}$. Density of the sphere model: $+1000 \mathrm{~kg} \mathrm{~m}^{-3}$. 
with parameters $\Delta x=\Delta y=200 \mathrm{~m}$ and $\Delta x=\Delta y=100 \mathrm{~m}$, using two typical interpolation methods: Kriging and Minimum Curvature. We can see that results for the Kriging method give results with quite well developed local minima in $\mathrm{L}_{1}$-norms (Fig. 5a and 5c), which can be used for source depth estimation (local minima are not so sharp as in Fig. 4a and 4b, but can be detected by the simple automatic 3-point operator). Second interpolation method (Minimum Curvature) did not work so well in this case (Fig. 5b and $5 \mathrm{~d})$.

Next trials work with the areal dimensions of interpreted grids (this is valid also for profile lengths). In general, it is valid (e.g. from the total normalized gradient method, Elysseiva and Pašteka, 2009) that in DC methods the dimension of the grid should be 5 to 10 times larger than the expected
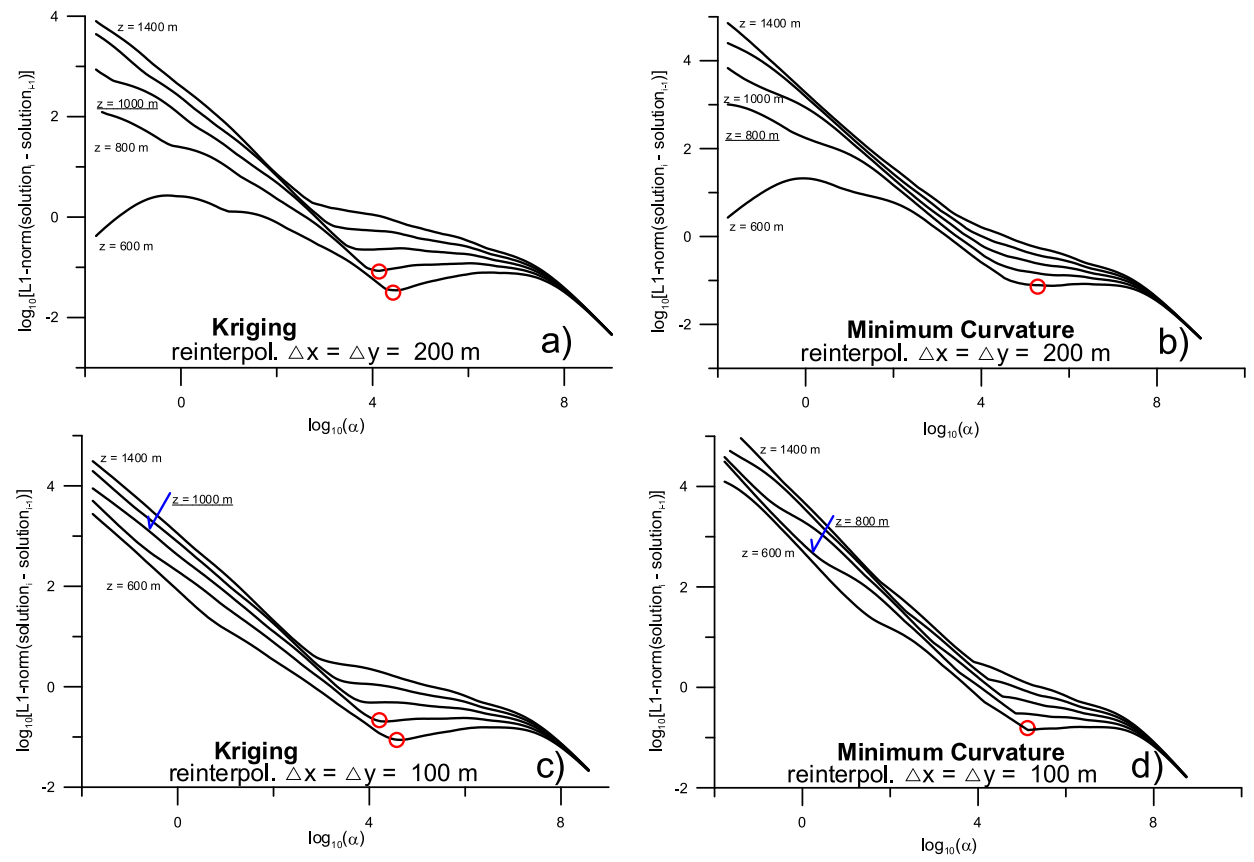

Fig. 5. Graphs of evaluated $\mathrm{L}_{1}$-norms for the sphere model (grid dimensions for all cases: $5 \times 5 \mathrm{~km}$ ) calculated for different sampling steps and different interpolation methods, used for re-gridding the original data: a) $\Delta x=\Delta y=200 \mathrm{~m}$ (Kriging method), b) $\Delta x=\Delta y=200 \mathrm{~m}$ (Minimum Curvature method), c) $\Delta x=\Delta y=100 \mathrm{~m}$ (Kriging method), d) $\Delta x=\Delta y=100 \mathrm{~m}$ (Minimum Curvature method). Density of the sphere model: $+1000 \mathrm{~kg} \mathrm{~m}^{-3}$. 
source depth. This is a quite large size and we cannot usually separate isolated anomalies with such a large (anomaly free) surroundings. But also here, there exist a solution, which helps sometimes. But let us start with our experiment results. In Fig. 6 we can see results for grids with 3 different areal dimensions: $2 \times 2 \mathrm{~km}, 3 \times 3 \mathrm{~km}$ and $4 \times 4 \mathrm{~km}$ (sampling step is quite detailed and identical for all of them: $\Delta x=\Delta y=100 \mathrm{~m}$ ). For smaller grid dimensions, the TRDC method works not well (Fig. 6b and 6d), local minima can be found almost on each depth level and the interpreted depth is over-estimated. For larger dimension, the method gives correct result (Fig. 6f) (here we can see that it was enough, when the grid size was 4 times larger than the source depth). Situation with small grid sizes can be quite hard for an unexperienced user, but the best way is to try several grid sizes and compare the results. Enlargement (expansion) of the grid can be realized by means of various extrapolation (padding) methods. We have tested few methods for the extrapolation of smaller grids to larger dimensions. We have realized that traditional interpolation methods (Kriging, Minimum Curvature, Radial Basis Functions, Inverse Distance method) are not suitable for larger expansions of grids (Fig. 7c and 7d), because there occurs always some kind of data deformation in the expanded grid. Also the well-known and used extrapolation approach - the cosine-taper padding method do not work properly for such large extrapolation of data (Fig. 7e). We have found a very interesting approach introducing 5 different numerical methods, coming from sparse linear algebra, which is used for interpolation and extrapolation of 2D digital data-sets (D'Errico, 2012; also published on Mathworks File Exchange). This approach can use various methods of approximation yielding a system of linear equations, solved then by means of sparse linear algebra methods (D'Errico, 2018, personal communication). User has to try several methods and then visually check, which is giving the best results. During performing experiments with data sets in the frame of this paper, we had best experiences with method nr. 3, which is based on partial differential equation approximation, using finite difference approximation. As we can see, extrapolated grid in Fig. $7 \mathrm{f}$ is in a very good agreement with the synthetic data, evaluated for the same area (Fig. 7b) here we have also used the mentioned third method. In Fig. 8 we can see two examples with expanded grids TRDC interpretation, when using again the third method (extrapolation to dimensions $3 \times 3 \mathrm{~km}$ and $4 \times 4 \mathrm{~km}$ ). Obtained 
results are very similar to that from synthetic data experiments (Fig. 6d and 6f). We can see that also here an acceptable result was obtained for the grid with larger dimensions $(4 \times 4 \mathrm{~km})$ - displayed C-norms show vanishing of the local minimum for the correct depth $=1000 \mathrm{~m}$ (Fig. 8d).
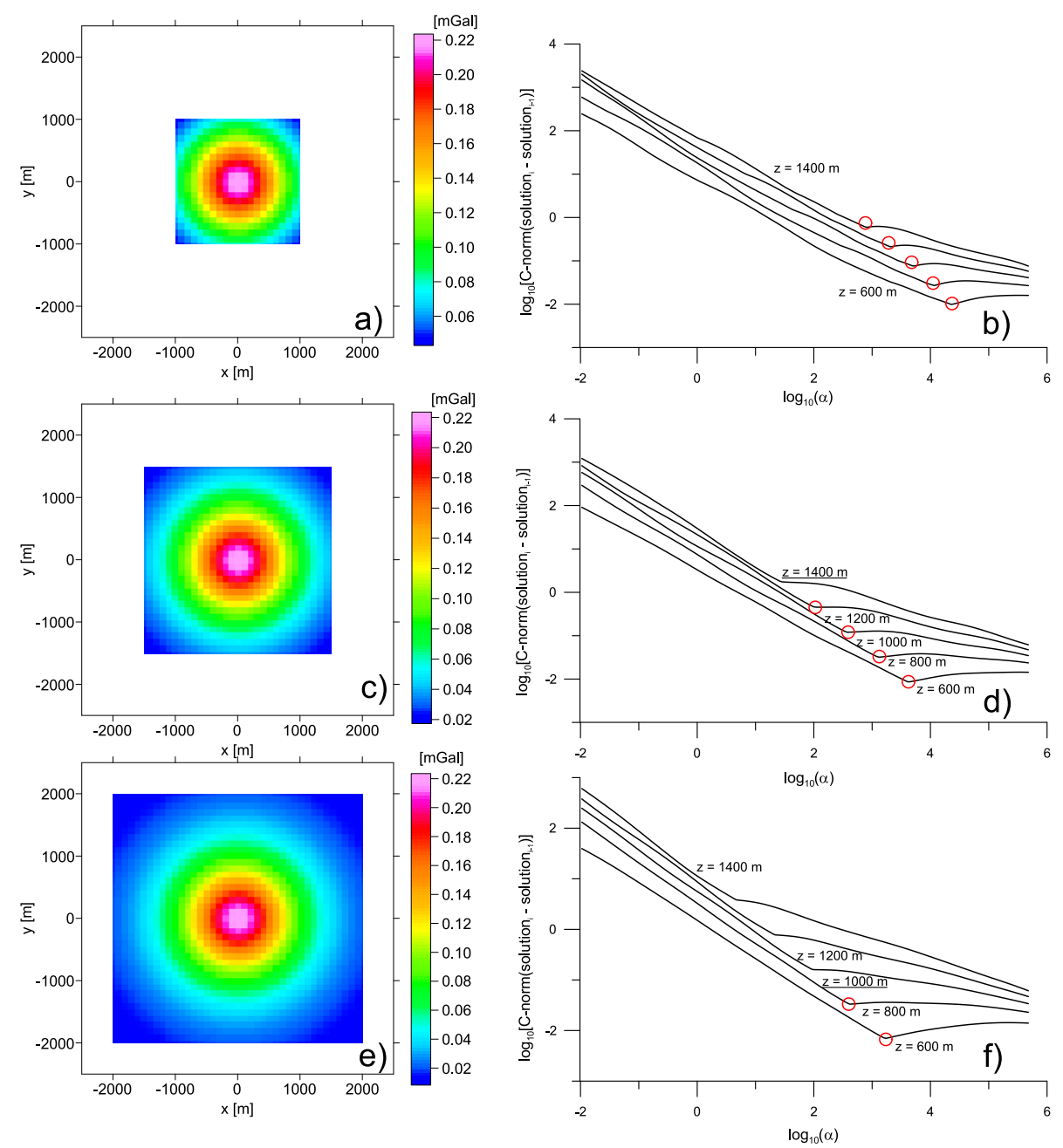

Fig. 6. Grids and graphs of evaluated C-norms for the sphere model (sampling steps for all cases: $\Delta x=\Delta y=100 \mathrm{~m}$ ) calculated for different dimensions of interpreted grids: a) $2 \times 2 \mathrm{~km}, \mathrm{~b}$ ) corresponding C-norms, c) $3 \times 3 \mathrm{~km}, \mathrm{~d}$ ) corresponding C-norms, e) $4 \times 4 \mathrm{~km}$, f) corresponding C-norms. Density of the sphere model: $+1000 \mathrm{~kg} \mathrm{~m}^{-3}$. 

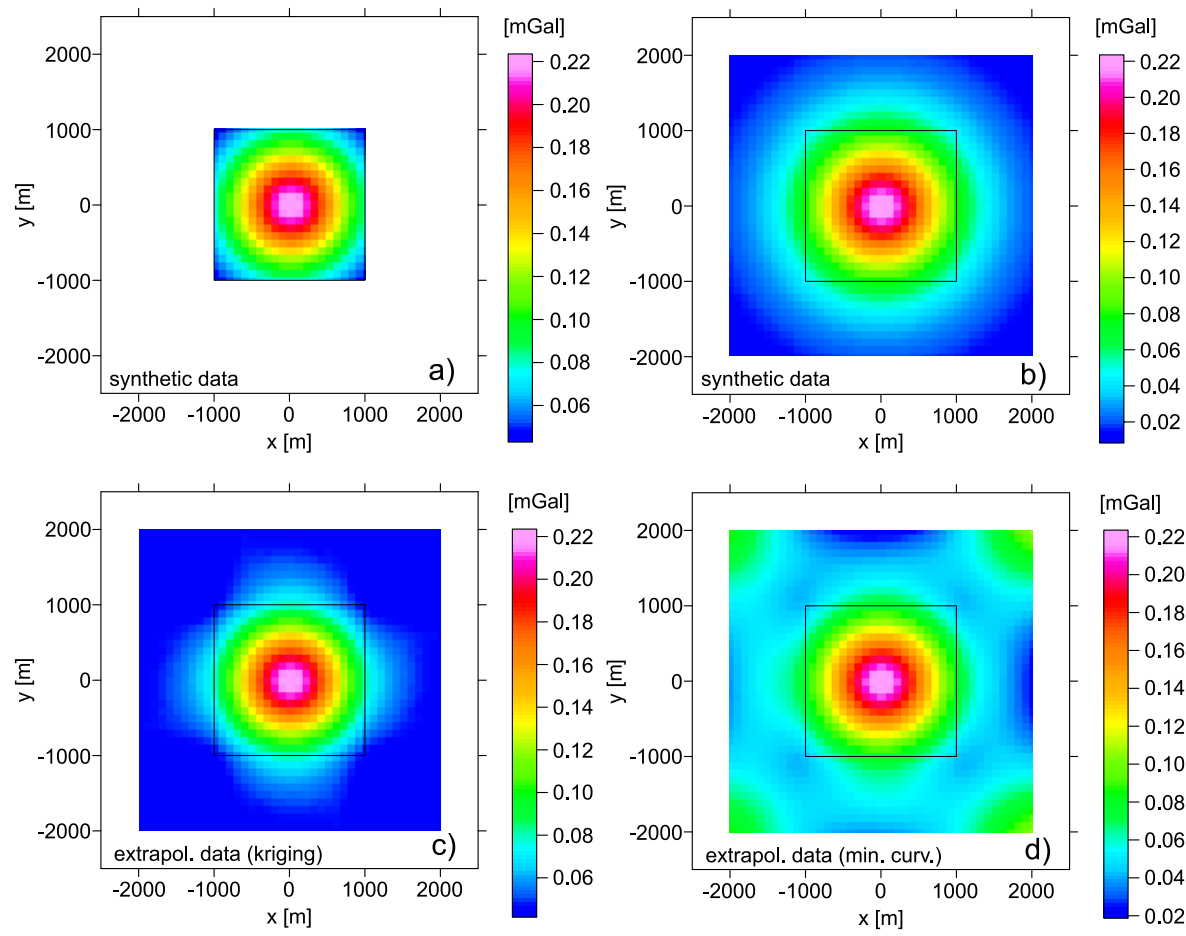

[mGal]
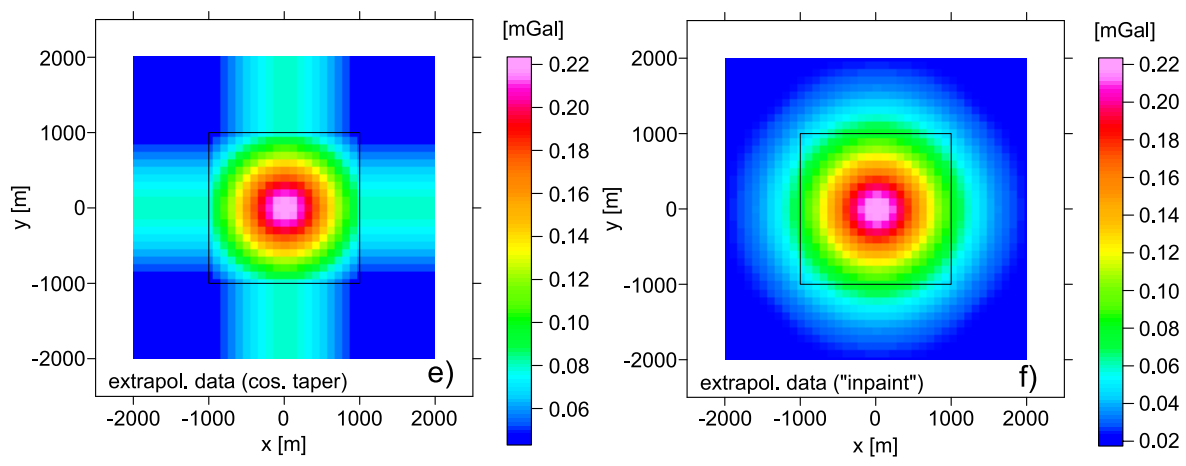

Fig. 7. Examples of expanded grids, using various extrapolation and padding methods, vertical component of gravitational acceleration field for a sphere: a) original synthetic data, grid with $2 \times 2 \mathrm{~km}$ dimension (used for extrapolation), b) synthetic data, larger grid with $4 \times 4 \mathrm{~km}$ dimensions, c) extrapolated grid to $4 \times 4 \mathrm{~km}$ (Kriging method), d) extrapolated grid to $4 \times 4 \mathrm{~km}$ (Minimum Curvature method), e) extrapolated grid to $4 \times 4 \mathrm{~km}$ (Cosine-taper method), f) extrapolated grid to $4 \times 4 \mathrm{~km}$ (partial differential equations approximation). Density of the sphere model: $+1000 \mathrm{~kg} \mathrm{~m}^{-3}$. 

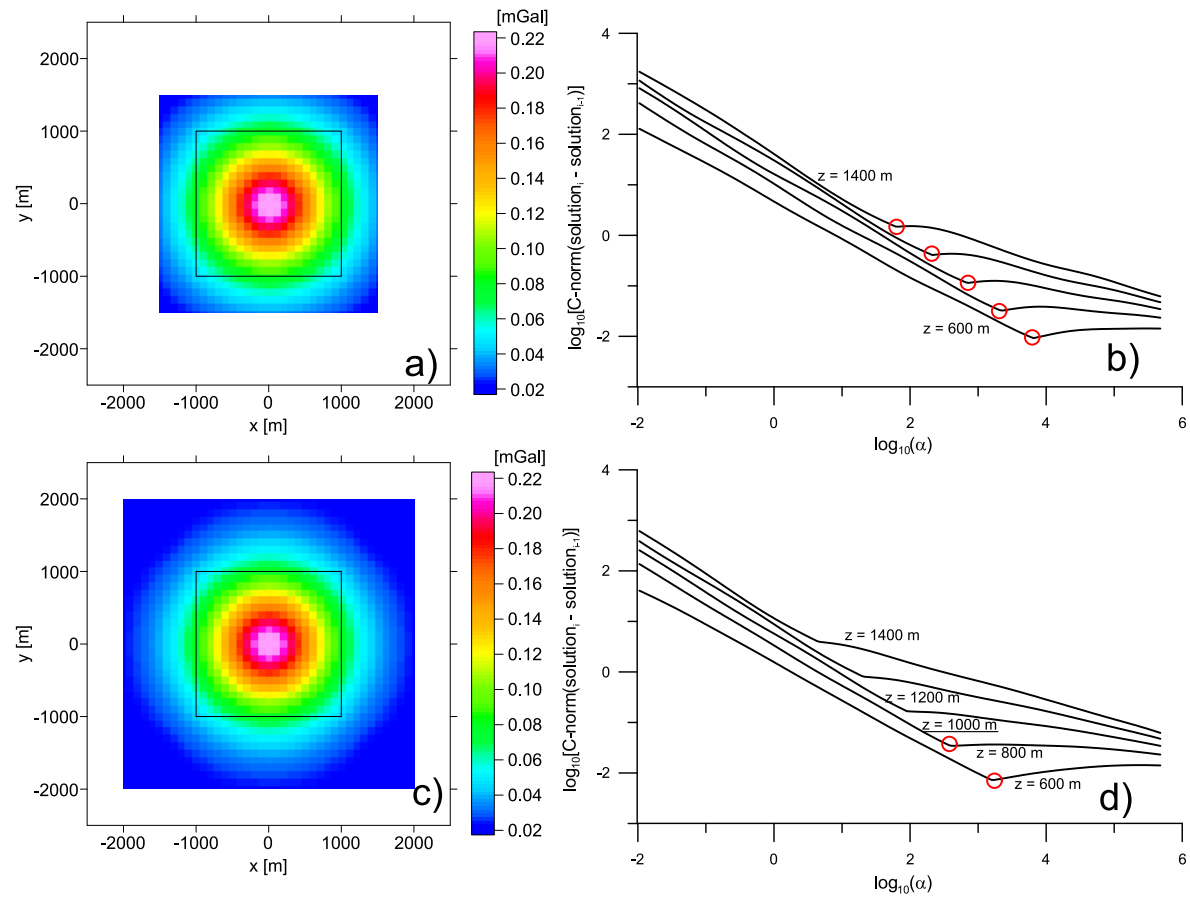

Fig. 8. Two examples of expanded grids (partial differential equations approximation) together with their corresponding C-norms: a) extrapolated to $3 \times 3 \mathrm{~km}$, b) corresponding C-norms, c) extrapolated to $4 \times 4 \mathrm{~km}, \mathrm{~d}$ ) corresponding C-norms. Sampling steps for all cases: $\Delta x=\Delta y=100 \mathrm{~m}$.

\section{Real world data interpretation - high definition UXO de- tection study}

As we have shown in our synthetic examples and as it follows from our experience with real data-sets interpretation, TRDC method is suitable for the interpretation of well developed and separable anomalies from isolated and compact source bodies. In near surface geophysical applications, the search for UneXploded Ordnance (UXO) object by means of high-definition magnetic survey produces often such kind of anomalies (interesting study of regularized DC algorithm application for UXO magnetic data interpretation is given by Li et al., 2013). Here we bring two examples from such a type of survey. In the first example the depth of the ordnance object was 
known and test measurements have been realized over it (ordnance was positioned at the earth surface). In the second example, we have selected one real anomaly from a performed UXO survey in SW Slovakia (site RohožníkStudienka), where the detected objects have been excavated by professional pyrotechnicians. In both examples, the amplitude of the total magnetic induction field $(T)$ was acquired by means of a Cs-vapour magnetometer with a sampling step along measured lines equal to $0.1 \mathrm{~m}$. Anomalous field $\Delta T$ was obtained by means of median filter application along measured lines. In the first example, the distance between the measured lines was $0.5 \mathrm{~m}$, in the second one it was $1.0 \mathrm{~m}$. In both examples the ordnance was a $100 \mathrm{~mm}$ diameter tank projectile.

In Fig. 9 we can see results from the first experiment. When a larger
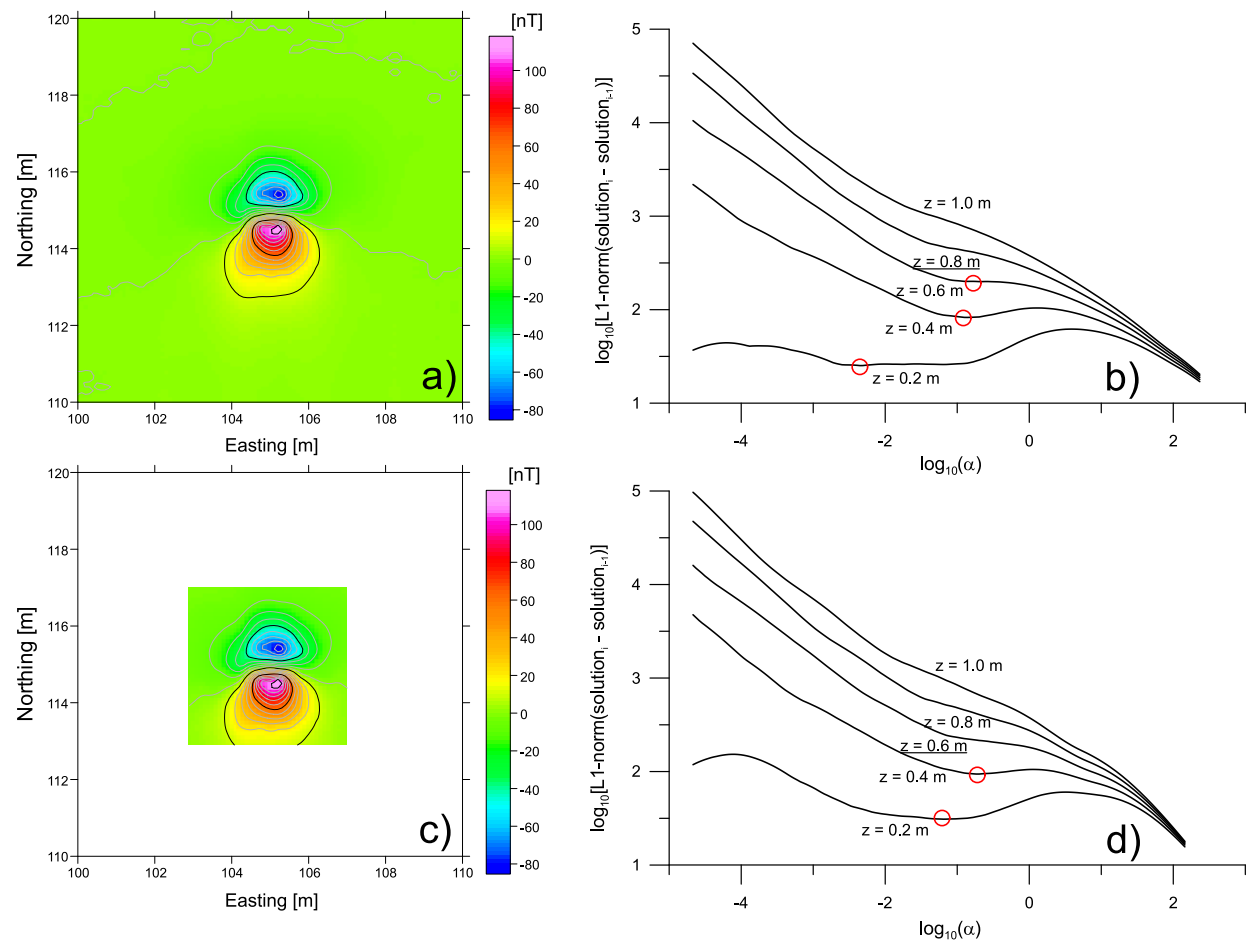

Fig. 9. Real world magnetic data-set, anomalous $\Delta T$ field over a $100 \mathrm{~mm}$ tank projectile, in the depth $1.0 \mathrm{~m}$ below the sensor: a) measured data on $10 \times 10 \mathrm{~m}$ area, b) corresponding $\mathrm{L}_{1}$-norms, c) extracted data on $3 \times 3 \mathrm{~m}$ area over the projectile, d) corresponding $\mathrm{L}_{1}$-norms. Sampling steps for all cases: $\Delta x=\Delta y=0.1 \mathrm{~m}$. 
surrounding of the anomaly has been selected $(10 \times 10 \mathrm{~m}$ area, Fig. 9a), then a larger depth of the estimated source was obtained $(0.8 \mathrm{~m})$ (Fig. 9b). When compared with the real depth $(1.0 \mathrm{~m})$, there is an error of $20 \%$, which is acceptable in this kind of depth-estimations. In the case of an extracted (smaller) grid $-(3 \times 3 \mathrm{~m}$ area, Fig. 9c), the estimated depth is shallower $(0.6 \mathrm{~m})$ (Fig. $9 \mathrm{~d})$. For the interpretation $\mathrm{L}_{1}$-norms have been selected, although shapes of their local minima do not have the perfect "sharp" shape (as we have seen in the case of synthetic data-sets interpretation). This is often the case, when we work with the real-world data-sets.
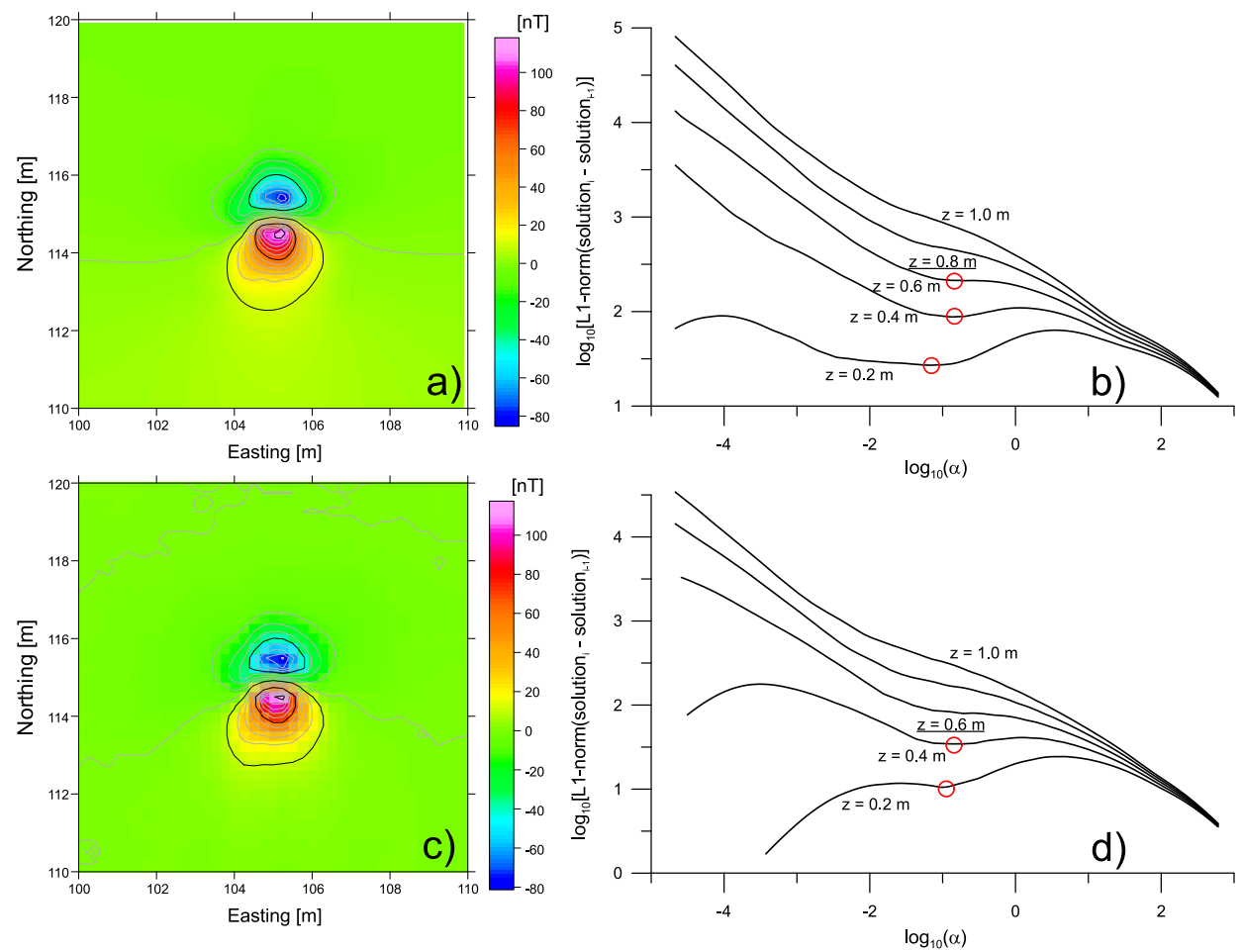

Fig. 10. Real world magnetic data-set, anomalous $\Delta T$ field over a $100 \mathrm{~mm}$ tank projectile, in the depth $1.0 \mathrm{~m}$ below the sensor: a) extrapolated data on $10 \times 10 \mathrm{~m}$ area (from $3 \times 3 \mathrm{~m}$ area in Fig. 9c by means of partial differential equations approximation), b) corresponding $\mathrm{L}_{1}$-norms, c) re-interpolated measured data on the original $10 \times 10 \mathrm{~m}$ area, d) corresponding $\mathrm{L}_{1}$-norms. Sampling steps: for case a): $\Delta x=\Delta y=0.1 \mathrm{~m}$ and for case c): $\Delta x=\Delta y=0.25 \mathrm{~m}$. 
In Fig. 10a we can see an extrapolated grid (with the same sampling step $\Delta x=\Delta y=0.1 \mathrm{~m}$ ), which was extrapolated from $3 \times 3 \mathrm{~m}$ area (Fig. 9c) to $10 \times 10 \mathrm{~m}$ area by means of the mentioned partial differential equations together with sparse linear algebra approach (D'Errico, 2012), using the method nr. 3. It can be easily seen that the L1-norms interpretation in the case of this extrapolated grid brought very similar results (Fig. 10b) like it was in the case of original data in Fig. (9b) - it resulted again to the depth of $0.8 \mathrm{~m}$. In Fig. 10c we show as an addition the effect of a coarse sampling step - the original data on the area of $10 \times 10 \mathrm{~m}$ have been re-grided with a sampling step $\Delta x=\Delta y=0.25 \mathrm{~m}$, which resulted again to a shallow depth of $0.6 \mathrm{~m}$ (Fig. 10d).

Final example comes from real UXO survey in an area close to an ac-
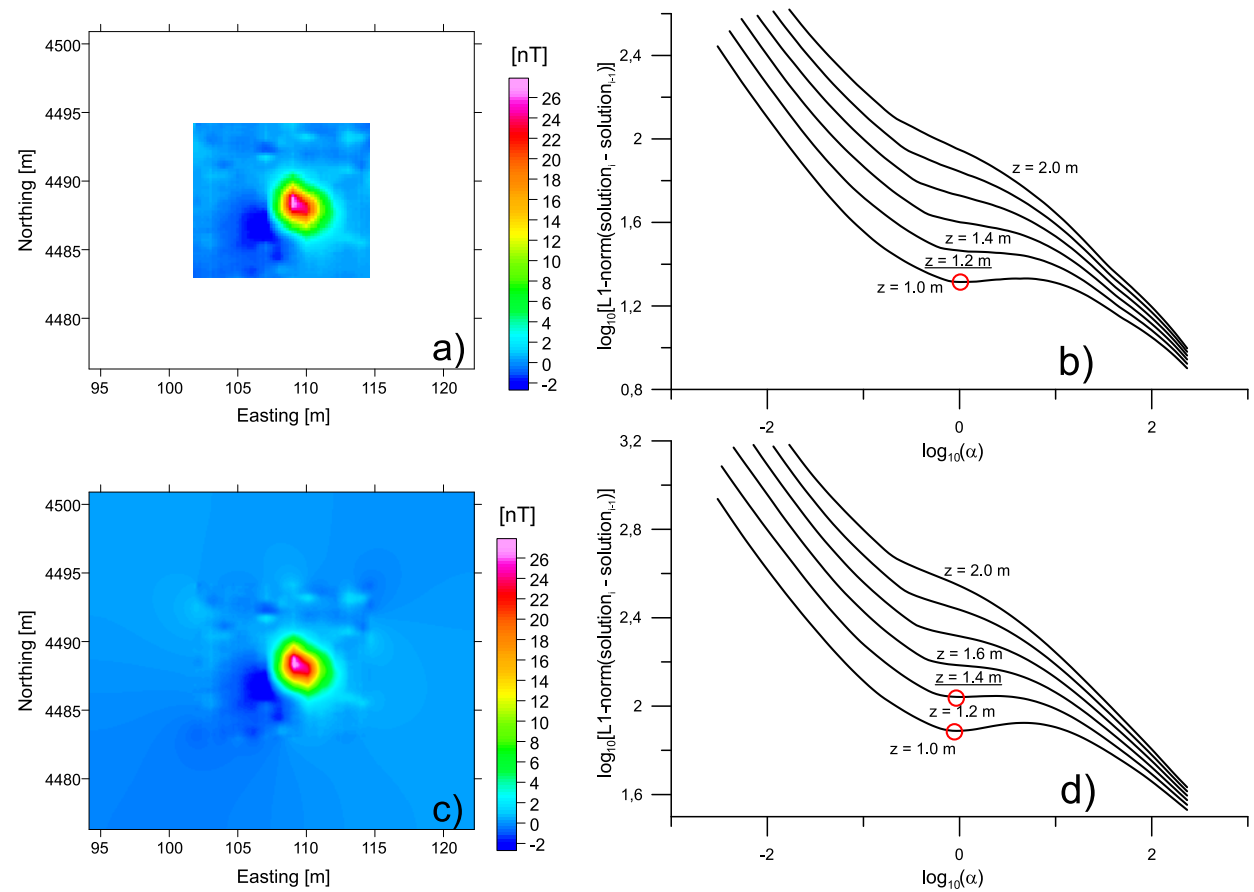

Fig. 11. Real world magnetic data-set, anomalous $\Delta T$ field over a $100 \mathrm{~mm}$ tank projectile, in the depth $1.9 \mathrm{~m}$ below the sensor: a) extracted measured data on $16 \times 15 \mathrm{~m}$ area, b) corresponding $\mathrm{L}_{1}$-norms, c) extrapolated data to $24 \times 23 \mathrm{~m}$ area, d) corresponding $\mathrm{L}_{1}$ norms. Sampling steps for all cases: $\Delta x=\Delta y=0.1 \mathrm{~m}$. 
tive shooting area in Rohožník-Studienka in SW Slovakia (survey was done because of planned vibroseismic survey in this area). As it was mentioned in the begin of this sub-chapter, magnetic data have been acquired in a net of $1.0 \times 0.1 \mathrm{~m}$ points. One typical anomaly was selected for TRDC depth-estimation (Fig. 11a). This anomaly is connected with an unexploded $100 \mathrm{~mm}$ tank gun projectile, which was excavated in the depth $1.3 \mathrm{~m}$ below the surface (the average height of the magnetometer sensor over the earth surface was around $0.6 \mathrm{~m}$ ). On the shapes of interpreted $\mathrm{L}_{1}$-norms (Fig. 11b) it can be seen that the detected local minimum vanishes too early and the estimated source depth $0.6 \mathrm{~m}$ is too shallow $(0.6 \mathrm{~m}=1.2 \mathrm{~m}-0.6 \mathrm{~m})$. In this case unfortunately, the extrapolation by means of the mentioned partial differential equations together with sparse linear algebra approach (D'Errico, 2012) did not helped much. Estimated depth in the case of extrapolated grid (Fig. 11c) is slightly larger (Fig. 11d), but its value $0.8 \mathrm{~m}$ $(1.4 \mathrm{~m}-0.6 \mathrm{~m})$ is in a large error, when compared with the real depth of the projectile $(1.3 \mathrm{~m})$. Here we can see that sometimes even such kind of extrapolation does not help and we have still to work on this aspect of the method (searching for better extrapolation methods).

\section{Conclusions}

Presented results show that during application of stable downward continuation methods (here the Tikhonov's regularization method was used) for the estimation of source depths also the discretization parameters of the interpreted fields can play an important role. The most important are the dimensions of the area covered by the interpreted field - in other words, there is a need for relatively large surroundings around the interpreted anomaly (from our experience these should be 5-10 times larger than the expected source depth). From this point of view, the method is more suitable for the interpretation of objects in near surface and mining geophysics (anomalies from cavities, UXO objects and ore bodies). Some extrapolation methods (based on partial differential equations approximation) can help in these situation. Beside of this, also the sampling step plays an important role we can improve the properties of the method even by introduction of interpolated values, covering the area in a denser grid. Although, we do not 
increase the information content of the anomalous field, but we improve the numerical aspects of the discrete Fourier transform evaluation, which is the basis of the method). From our experiences, at least 10 sampling points pro anomaly half-with should represent a material, which is good for the interpretation with this kind of methods. In this situations, the well know Kriging interpolation method gives the best results.

Together with this paper, we give a software solution of this proposed and analysed method - the Matlab script REGCONT2 is free for academic and scientific use and it can be downloaded together with supporting files and a user manual from the server: http://www.kaeg.sk/vyskum/projekt-vega-20 14/project-vega-1-0462-16/.

Acknowledgements. Authors would like to express their thanks to Dr. Katarina Brazda from Vienna University and Dr. Peter Fid'o Richter for an inspiration in the derivation of basic spectral characteristics of the method, given in the paper Appendix. Thanks belong also to the company G-trend Ltd. Bratislava for allowing to publish the UXO magnetometric data from site Rohožník-Studienka. Published research was supported by the Slovak scientific agencies, projects VEGA 1/0141/15, VEGA 1/0462/16 and APVV-16-0146. Some aspects have been solved also a part of the scientific program in the project: Comenius University Science Park supported by the Research and Development Operational Programme funded by the ERDF Grant number ITMS 26240220086. Authors are thankful for valuable comments and suggestions, obtained in the frame of the review process.

\section{References}

Abedi M., Gholami A., Norouzi G. H., 2014: A new stable downward continuation of airborne magnetic data based on Wavelet deconvolution. Near Surface Geophysics 12, 751-762.

Bath M., 1968: Mathematical aspects of seismology. Elsevier, 428 p.

Baranov W., 1975: Potential fields and their transformation in applied geophysics. Gebruder Borntraeger, Berlin-Stuttgart, $151 \mathrm{p}$.

Blakely R. J., 1995: Potential Theory in Gravity and Magnetic Applications. Cambridge University Press, UK, $441 \mathrm{p}$.

Cooper G., 2004: The stable downward continuation of potential field data. Explor. Geoph., 35, 4, 260-265.

Cressie N. A. C., 1991: Statistics for Spatial Data. John Wiley and Sons, Inc., New York, $900 \mathrm{p}$. 
D'Errico J., 2012: Interpolation (\& extrapolation) of NaN elements in a $2 \mathrm{~d}$ array inpaint_nans Matlab function. (https://de.mathworks.com/matlabcentral/file exchange/4551-inpaint_nans), MATLAB Central File Exchange. Retrieved October 10, 2017.

Elysseieva I. S., Pašteka R., 2009: Direct interpretation of 2D potential fields for deep structures by means of the quasi-singular points method. Geophysical Prospecting, $\mathbf{5 7}, 4,683-705$.

Fedi M., Florio G., 2002: A stable downward continuation by using the ISVD method. Geophysical Journal International 151, 146-156.

Florio G., Fedi M., 2018: Depth estimation from downward continuation: An entropybased approach to normalized full gradient. Geophysics 83, 3, J33-J42.

Glasko V. B., Litvinenko O. K., Melikhov V. R.,1970: Possibilities of regularizing algorithms for continuation of potential functions close to source masses. Prikladnaya Geofizika (Applied Geophysics) 60, 142-157 (in Russian).

Hansen P. C., 2007: Regularization Tools Version 4.0 for Matlab 7.3. Numerical Algorithms 46, 189-194.

Lawson C. L., Hanson R. J., 1974: Solving Least Squares Problems. Prentice-Hall, Englewood Cliffs, 305 p.

Li Y., Devriese S. G. R., Krahenbuhl R. A., Davis K., 2013: Enhancement of magnetic data by stable downward continuation for UXO application. IEEE Transactions on Geoscience and Remote Sensing, 51, 6, 3605-3614.

Ma G., Liu C., Huang D., Li L., 2013: A stable iterative downward continuation of potential field data. Journal of Applied Geophysics 98, 205-211.

Mudretsova E. A., Veselov K. E., Ed., 1990: Gravimetry. Nedra, Moscow, 607 p. (in Russian).

Pašteka R., Karcol R., Pašiaková M., Pánisová J., Kušnirák D., Beres J., 2011: Depth estimation of microgravity anomaliessources by means of regularized downward continuation and Euler deconvolution. 73rd EAGE Conference and Exhibition extended abstract, p. 1-5.

Pašteka R., Karcol R., Kušnirák D., Mojzeš A., 2012: REGCONT: A Matlab based program for stable downward continuation of geophysical potential fields using Tikhonov regularization. Computers and Geosciences, 49, 278-289.

Smith W. H. F., Wessel P., 1990: Gridding with continuous curvature splines in tension. Geophysics, 55, 3, 293-305.

Tikhonov A. N., Glasko V. B., Litvinenko O. K., Melichov V. P., 1968: Analytic continuation of a potential to disturbing masses by the regularization method. Izv., Earth Physics, 12, 738-747.

Trompat H., Boschetti F., Hornby P., 2003: Improved downward continuation of potential field data. Exploration Geophysics 34, 4, 249-256.

Troutman J. L., 1983: Variational Calculus with Elementary Convexity. Springer, 364 p.

Zeng X., Li X., Su J., Liu D., Zou H., 2013: An adaptive iterative method for downward continuation of potential-field data from a horizontal plane. Geophysics, 78, 4, J43-J52. 
Zeng X., Liu D., Li X., Chen D., Niu C., 2014: An improved regularized downward continuation of potential field data. Journal of Applied Geophysics, 106, 114-118.

Zeng X., Li X., Jia W., Chen D., Liu D., 2015: Iterative Wiener filter for unstable linear transformations of potential field data. Journal of Applied Geophysics, 115, 100109.

Zhang H., Ravat D., Hu X., 2013: An improved and stable downward continuation of potential field data: The truncated Taylor series iterative downward continuation method. Geophysics, 78, 5, J75-J86.

Zhang Y., Wong Y. S., Lin Y., 2016: BTTB-RRCG method for downward continuation of potential field data. Journal of Applied Geophysics, 126, 74-86.

Zhang C., Lü Q., Yan J., Qi G., 2018: Numerical Solutions of the Mean-Value Theorem: New Methods for Downward Continuation of Potential Fields. Geophysical Research Letters, 45, 8, 3461-3470.

Zhou S., Li J., Yuan Y., 2018: Downward Continuation of Potential Field Data Based on Chebyshev-Padé Approximation Function. Pure and Applied Geophysics, 175, 1, $275-286$.

\section{Appendix}

\section{Derivation of the regularized downward continuation filter in Fou- rier domain}

This derivation was inspired by the fundamental paper from Tikhonov et al. (1968) and we try to give it here with all needed details (for better understanding of the basic idea of regularization).

Based on the harmonic property of the continued potential field function $U$ (potential or its higher derivative; which fulfils the Laplace's equation) and Green's third identity, the so-called Poisson's integral is valid for the analytical continuation (e.g. Blakely, 1995, p. 316):

$U(x, y, z-\Delta z)=\frac{\Delta z}{2 \pi} \int_{-\infty}^{\infty} \int_{-\infty}^{\infty} \frac{U(\xi, \eta, z)}{\left[(x-\xi)^{2}+(y-\eta)^{2}+\Delta z^{2}\right]^{3 / 2}} \mathrm{~d} \xi \mathrm{d} \eta$

where $U(x, y, z-\Delta z)$ is the potential field on a depth level $h-\Delta z$ (further from the sources), $U(x, y, z)$ is the field on the level $z$ (closer to the sources), $\Delta z$ is the vertical continuation distance $(\Delta z>0 ; z$-axis is pointing downwards) and $\xi, \eta$ are the equivalents of variables $x, y$ in the integration plane. When the function $U(x, y, z-\Delta z)$ is searched then we speak about 
an upward and when $U(\xi, \eta, z)$ inside the integral then about a downward continuation, respectively. Upward continuation is a stable operation (it is an integral transformation) and downward continuation is an unstable operation (it must be searched as a solution of an integral equation).

In the case of a $2 \mathrm{D}$ problem we can write:

$U(x, z-\Delta z)=\frac{\Delta z}{\pi} \int_{-\infty}^{\infty} \frac{U(\xi, z)}{\left[(x-\xi)^{2}+\Delta z^{2}\right]} \mathrm{d} \xi . \quad$ (2D problem)

Equation (A.2) (and A.1 of course too) can be seen as a convolution integral: e.g. for the 2D problem it can be rewritten as $U(x, y, z-\Delta z)=U(x, z) \otimes$ $K(x)$, where $\otimes$ is the convolution symbol and $K(x)=(1 / \pi)\left(\left(\Delta z /\left(x^{2}+\right.\right.\right.$ $\left.\Delta z^{2}\right)$ ) is the integral kernel function from (A.2). Convolution operation can be performed effectively in the Fourier domain using the convolution theorem, what will be used later on.

The most important aspect of the regularized solution creation in the sense of Tikhonov approach (Tikhonov et al., 1968; Tikhonov and Arsenin $1977)$ can be defined as a minimization problem solution - we have to minimize a functional $(F)$, which is composed by two partial functionals (objective functions). The first of them is describing the closeness of the searched regularized solution to classical one (the searched function is continued upwards and compared with the original measured function); the second one is the regularizing (smoothing) functional, assuming the fact that a sum of the squared horizontal derivative of the searched solution should be as small as possible. The aim is to find a solution, which will be in a kind of "equilibrium" between these two conditions.

In the next part of the text we will derive, based on this minimization scheme, the solution for the downward continuation (Tikhonov et al., 1968). Because of the lack of simplicity we will derive it for the 2D problem, taking the starting level at $z=0, U_{0}=U(x, 0)$ and the downward continuation will be performed to the depth level $h(\Delta z=h), y(x)=U(x, h)$. The minimization problem can be formulated as:

$\int_{-\infty}^{\infty} F\left[y, y^{\prime}, x\right] \mathrm{d} x=\int_{-\infty}^{\infty}\left\{\left[y \otimes K(x)-U_{0}(x)\right]^{2}+\alpha\left[y^{\prime}(x)\right]^{2}\right\} \mathrm{d} x=\min$. 
where $y^{\prime}(x)$ stands for the horizontal derivative of $y$ with respect to $x(\partial y / \partial x)$ and $\alpha$ is the regularization parameter "managing" the influence of the second (stabilizing) functional upon the whole solution (physical unit of $\alpha$ is $\left.\mathrm{m}^{-1}\right)$.

The aim of the solution of this minimization problem is to find the solution $y(x)$, for which the functional $F[$ ] reaches a minimum. We use the known Euler-Lagrange equation from the variational calculus theory (e.g. Bath, 1968; Troutman, 1983) for finding an extreme of the variational problem:

$\frac{\partial F}{\partial y}-\frac{\mathrm{d}}{\mathrm{d} x}\left[\frac{\partial F}{\partial y^{\prime}}\right]=0$,

The first term of the Euler-Lagrange equation (A.4) is:

$$
\begin{aligned}
\frac{\partial F}{\partial y} & =2\left(y \otimes K-U_{0}\right) \frac{\partial(y \otimes K)}{\partial y}=2\left(y \otimes K-U_{0}\right) \int_{-\infty}^{+\infty} K(x-\xi) \mathrm{d} \xi= \\
& =2\left(y \otimes K-U_{0}\right) \frac{1}{\pi} \int_{-\infty}^{+\infty} \frac{h}{(x-\xi)^{2}+h^{2}} \mathrm{~d} \xi=2\left(y \otimes K-U_{0}\right),
\end{aligned}
$$

where the incident integral of the shifted kernel function is equal to 1 (with assumption $h>0$ ).

The second term of the Lagrange-Euler equation (A.4) is:

$\frac{\mathrm{d}}{\mathrm{d} x}\left[\frac{\partial F}{\partial y^{\prime}}\right]=\frac{\mathrm{d}}{\mathrm{d} x}\left[2 \alpha y^{\prime}\right]=2 \alpha y^{\prime \prime}$.

These results are substituted to equation (A.4) and we yield:

$2 y \otimes K-2 U_{0}-2 \alpha y^{\prime \prime}$.

This equation is solved in spectral domain by using theorems of spectrum of convolution and theorem of spectrum of differentiation of a function:

The Fourier transformations $F$ of single terms are ( $u$ is the wave-number):

$F\{K(x)\}=\mathrm{e}^{-|u| h}-$ spectral characteristics of upward continuation $(h>0)$, $F\{y(x)\}=\tilde{y}(\mathbf{u})$ - spectrum of the solution (regularized downward continued field), 
$F\left\{y^{\prime \prime}(x)\right\}=-u^{2} \tilde{y}(u)-$ spectral characteristic of second horizontal derivative of the solution,

$F\left\{U_{0}(x)\right\}=\tilde{U}_{0}(u)$ - spectrum of measured field on the level $z=0$.

Substituting these terms into the equation (A.5) gives (divided by factor 2):

$\mathrm{e}^{-|u| h} \tilde{y}-\tilde{U}_{0}-\alpha\left(-u^{2}\right) \tilde{y}=0$,

$\tilde{y}\left[\mathrm{e}^{-|u| h}+\alpha u^{2}\right]=\tilde{U}_{0}$,

$\tilde{y}\left[1+\alpha u^{2} \mathrm{e}^{|u| h}\right]=\mathrm{e}^{|u| h} \tilde{U}_{0}$.

The spectrum of searched solution is then equal:

$\tilde{y}=\frac{1}{1+\alpha u^{2} \mathrm{e}^{|u| h}} \mathrm{e}^{|u| h} \tilde{U}_{0}$,

where the first part (fraction) is a low-pass filter - the regularization term (controlled by the regularization parameter $\alpha$ ), the second one $\exp (|u| h)$ is the spectral characteristics of the classical downward continuation and the third is the spectrum $\tilde{U}_{0}(u)$ of continued function $U_{0}(x)$. A part of the regularized solution is the search for an optimum value of the regularization parameter $\alpha$ (there exist several methods, in this paper we use the concept of C-norms, or in general $\mathrm{L}_{\mathrm{P}}$-norms). 